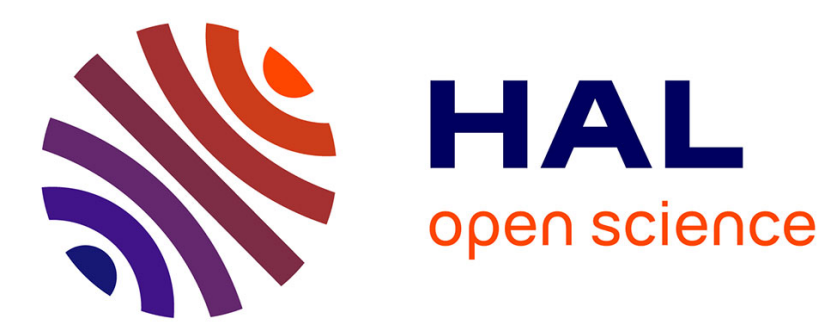

\title{
Data-Driven Respiration-Gated SPECT for Liver Radioembolization
}

Antoine Robert, Simon Rit, Thomas Baudier, Julien Jomier, David Sarrut

\section{To cite this version:}

Antoine Robert, Simon Rit, Thomas Baudier, Julien Jomier, David Sarrut. Data-Driven RespirationGated SPECT for Liver Radioembolization. IEEE Transactions on Radiation and Plasma Medical Sciences, 2022, 6 (7), pp.778-787. 10.1109/TRPMS.2021.3137990 . hal-03515752

\section{HAL Id: hal-03515752 https://hal.science/hal-03515752}

Submitted on 6 Jan 2022

HAL is a multi-disciplinary open access archive for the deposit and dissemination of scientific research documents, whether they are published or not. The documents may come from teaching and research institutions in France or abroad, or from public or private research centers.
L'archive ouverte pluridisciplinaire HAL, est destinée au dépôt et à la diffusion de documents scientifiques de niveau recherche, publiés ou non, émanant des établissements d'enseignement et de recherche français ou étrangers, des laboratoires publics ou privés. 


\title{
Data-Driven Respiration-Gated SPECT for Liver Radioembolization
}

\author{
Antoine Robert, Simon Rit, Thomas Baudier, Julien Jomier, and David Sarrut
}

\begin{abstract}
Respiratory motion leads to blur and artifacts in single photon emission computed tomography (SPECT) images. This may be an issue in some treatments such as liver radioembolization. The purpose of this work was to reconstruct four-dimensional (4D) respiration-gated SPECT images using a data-driven approach. A respiratory signal was extracted from SPECT list-mode data using Laplacian Eigenmaps and used for retrospective phase gating. The method was evaluated on various SPECT datasets: Monte Carlo simulation of a patient acquisition, several acquisitions of a dynamic phantom and 20 acquisitions of patients treated with liver radioembolization. We observed an increase of the mean activity inside the tumor (A) and of the tumor-to-normal liver ratio $(T / N)$ in respiration-gated SPECT image compared to the 3D image without motion correction. For the patient acquisitions, an average $3.3 \%$ (up to $11.9 \%$ ) and $3.2 \%$ (up to $9.7 \%$ ) increase was observed for $A$ and $T / N$, respectively. For the simulation, the activity was within $2.6 \%$ of the reference without movement and around $3 \%$ for the three patterns of tumor motion simulated with the mechanical phantom. Overall, the proposed respiration-gated SPECT reconstruction improves the estimation of the activity in the tumor without the need for extra hardware.
\end{abstract}

Index Terms-SPECT, breathing motion, radioembolization, tomographic reconstruction.

\section{INTRODUCTION}

$\mathbf{S}$ INGLE photon emission computed tomography (SPECT) is a key tool for imaging cancer, both for diagnosis and therapy monitoring purposes. One challenge is breathing motion which impacts the image quality by blurring the SPECT image. Indeed, the breathing period is of the order of a few seconds while the acquisition is of several minutes. For example, Bastiaannet et al [1] showed using simulated data that an underestimation of almost $30 \%$ in activity can be found using the SPECT images in liver tumors, compared to acquisitions without movement.

In early studies, efforts have been made to account for the movements of the patient during SPECT acquisitions [2][4] and for the heart motion in SPECT myocardial perfusion imaging [5], [6]. In order to handle breathing motion, a classical method is respiration-gated reconstruction. This method relies on the quasi-periodicity of the breathing, meaning that the spatial position of the patient is the same from cycle to

A. Robert is with Univ.Lyon, INSA-Lyon, Université Claude Bernard Lyon 1, UJM-Saint Etienne, CNRS, Inserm, CREATIS UMR 5220, U1206, F-69373, Lyon, France and Kitware SAS, 6 Cours André Philip, 69100 Villeurbanne.

S. Rit and D. Sarrut are with Univ.Lyon, INSA-Lyon, Université Claude Bernard Lyon 1, UJM-Saint Etienne, CNRS, Inserm, CREATIS UMR 5220, U1206, F-69373, Lyon, France.

T. Baudier is with Centre Lon Brard, 28, rue Laennec, 69373 Lyon Cedex 08, France.

J. Jomier is with Kitware SAS, 6 Cours André Philip, 69100 Villeurbanne. cycle and that this position is characterized by a respiratory signal. The method consists in splitting the respiratory cycle into several phases and using this signal to sort the measured projections according to these phases [7]-[10].

There are two families of methods to extract a respiratory signal. Hardware-based methods rely on an external sensor measuring a surrogate signal, e.g., the abdomen motion [11], [12] or the volume of air inhaled and exhaled [13]. Even though these approaches have been proven effective, they require extra hardware synchronized with the SPECT scanner. Furthermore, these surrogate signals may not reflect the internal motion [14], [15]. For these reasons, data-driven methods have been investigated to extract a respiratory signal from listmode data [16]-[20].

Most of the studies dealing with respiratory motion correction in nuclear imaging have been dedicated to positron emission tomography (PET) [19], [21]-[23]. In SPECT, respirationgated reconstruction has focused on myocardial perfusion imaging (MPI) [7], [24], [25] using extra-hardware to get a respiratory signal. Some studies investigating respiratory-gated SPECT of the lungs have also been reported [26], [27].

The recent emergence of targeted radionuclide therapies that use SPECT images for dose planning or monitoring, such as liver radioembolization or neuroendocrine tumors treated with ${ }^{177} \mathrm{Lu}$, increased the need for quantitative SPECT. Typically, one key step in liver radioembolization is the pre-treatment ${ }^{99 m} \mathrm{Tc}$ - MAA SPECT/CT acquisition used to assess lung shunt and extrahepatic uptake. This acquisition can also be used to prescribe the injected activity of therapeutic ${ }^{90} \mathrm{Y}$ microspheres and predict the 3D spatially distributed absorbed dose. The accuracy of this patient-specific treatment planning directly depends on these pre-treatment SPECT images.

In this work, we investigated respiration-gated SPECT reconstruction using a respiratory signal extracted from listmode data. The method was evaluated on both simulated and real SPECT data of a physical dynamic phantom and of patients treated with liver radioembolization.

\section{MATERiAls AND METHODS}

\section{A. Breathing signal extraction and gating}

A breathing signal was extracted from the list-mode data using the method based on the Laplacian Eigenmaps algorithm described by Sanders et al [18]. Unlike principal component analysis [19], Laplacian Eigenmaps take into account the intrinsic geometry of the data by building a graph accounting for spatial neighbors. The dimensionality reduction is done by minimizing a cost function based on the graph to ensure that points close to each other in the high-dimensional space 
are mapped close to each other in the low-dimensional space, making this method robust to noise.

The SPECT/CT system used for this study allowed us to acquire two projections simultaneously for each camera stop $p$ and to record the results in list-mode format for applying the Laplacian Eigenmaps algorithm as described in [18] and summarized in the following. The list-mode dataset, containing the detected counts with the spatial position, the energy and the timestamp of each photon impinging on the detector, was binned into $256 \times 256$ pixel projections at a high framerate $(5 \mathrm{~Hz})$ without predefining any target region. For each camera stop $p$, the two projections were concatenated and vectorized to yield a matrix $X_{p}=\left[x_{1}, x_{2}, \ldots, x_{T}\right]$ with $x_{i} \in \mathbb{R}^{2^{17}}$ corresponding to the input data at time bin $i$ and $T$ the number of $200 \mathrm{~ms}$ frames during the $p$-th camera stop. An affinity matrix $W$ was then computed to favor smooth respiratory signals:

$$
w_{i, j}=e^{-\frac{\left\|x_{i}-x_{j}\right\|_{2}^{2}}{\alpha}} \text { with } \alpha=\frac{1}{T^{2}} \sum_{i, j}\left\|x_{i}-x_{j}\right\|_{2}^{2} .
$$

The degree matrix $D$, which is a diagonal matrix that represents the degree of connectivity of each input data point, was derived from $W$ :

$$
d_{i, i}=\sum_{j} w_{i, j}
$$

The Laplacian $L=D-W$ was computed and the Laplacian Eigenmaps algorithm consists in solving the generalized eigenvalue problem:

$$
L y_{k}=\lambda D y_{k}
$$

where $y_{k}$ is the eigenvector that corresponds to the $\mathrm{k}$-th smallest non-zero eigenvalue $\lambda$. A vector $y_{p}=\left[y_{1}, y_{2}, \ldots, y_{T}\right]$ corresponding to the internal respiratory signal was obtained for each camera stop $p$ by taking the eigenvector associated to the smallest non-zero eigenvalue. Smoothing, polarity correction, baseline correction and normalization were then applied to each of the individual $y_{p}$ as described in [18].

Following [28], the end-inhale positions were detected by finding the minima on the local phase of the discrete-time analytic representation of the extracted signal [29]. Then, the breathing phase was computed by assuming linearity from $0 \%$ to $100 \%$ between two consecutive end-inhale positions. Measured projections were sorted into temporal bins of $12.5 \%$ phase width for all the acquisitions.

\section{B. SPECT reconstruction}

All volumes were reconstructed into a $128^{3}$ voxel matrix (voxel size $4.42 \mathrm{~mm}$ ) using the Reconstruction Toolkit (RTK) [30] with 20 iterations and 4 subsets of ordered-subsets expectation maximization (OSEM) algorithm with a quadratic penalty [31]. Scatter was corrected using a double energy window [32]. Point spread function (PSF) and attenuation were accounted for during the iterative process using the method of Zeng et al [33] with a free-breathing 3D CT image for all images.

For the 3D reconstruction, every eighth count was kept in the list-mode data to reconstruct a set of eight 3D images with the same level of noise as each frame of the 4D image.

\section{SPECT simulation using GATE}

A complete SPECT acquisition of a breathing patient was simulated with a Monte Carlo technique using GATE [34]. The simulated SPECT scanner was the same as the one of the phantom and patient acquisitions (section II-D). A 4D CT image of a thoracic patient made of 10 breathing phases was used as input of the simulation. The 4D CT image was obtained from a retrospective gating of a supine patient acquisition during free-breathing monitored by a respiratory belt. Deformable image registration was used to retrieve the deformation vector field between the reference end-exhale phase and each other phase. The emission map was made of a spheroid tumor of $20 \mathrm{~mm}$ radius positioned in the liver, approximately $40 \mathrm{~mm}$ below the diaphragm, with a ratio of 9 to 1 between the activity in the tumor and the liver background. In order to smooth the motion, the deformation fields were interpolated between consecutive phases and applied to the reference phase of the 4D CT and the emission map to obtain 20 frames. The motion of the tumor center had a peak-to-peak amplitude of $15 \mathrm{~mm}$ in the superior-inferior direction and 10 $\mathrm{mm}$ in the antero-posterior direction.

The parameters of the GATE simulation are given in [35]. The ${ }^{99 m}$ Tc was used as radionuclide and $6 \times 10^{9}$ primary particles were simulated. Each position of the tumor was simulated individually, resulting in twenty list-mode files of a complete gantry rotation. The events stored in those list-mode files were then merged into a single list-mode file according to the input respiratory signal to mimic one SPECT acquisition of a breathing patient. The input respiratory signal was a $\cos ^{4}$ function of $4 \mathrm{~s}$ period.

\section{Phantom and patient acquisitions}

Both the phantom and the patient datasets were acquired with the GE Discovery NM/CT 670 at the Léon Bérard cancer center. ${ }^{99 m} \mathrm{Tc}$ was used as radioisotope with the Low Energy High Resolution (LEHR) collimator. Sixty projections were acquired over $360^{\circ}$, each with $128 \times 128$ pixels, $4.42 \mathrm{~mm}$ isotropic spacing and an acquisition duration of $25 \mathrm{~s}$. Thanks to the dual-head SPECT system, two opposite projections were measured at the same time reducing the overall acquisition duration by a factor 2. Following the SPECT acquisitions, free breathing 3D CT images were acquired for attenuation correction.

The dynamic thorax phantom (CIRS, Norfolk, VA) was used as an anthropomorphic phantom with a tumor moving in the lungs. The tumor was modeled with an $8 \mathrm{ml}$ spherical insert filled with $54.9 \mathrm{MBq}$ of ${ }^{99 m} \mathrm{Tc}$. Four scans were acquired, one without motion and three with various tumor motions in the cranio-caudal direction. The tumor motion was first controlled with a $\cos ^{4}(2 \pi t / \mathrm{T})$ function of period $\mathrm{T}=4 \mathrm{~s}$ and peak-to-peak amplitudes of $10 \mathrm{~mm}$ and $20 \mathrm{~mm}$. The third motion pattern was extracted from a patient cone-beam CT acquisition in [36] (Fig. 1). The duration of the cone-beam CT acquisition (2 min) being shorter than that of a SPECT acquisition (14 $\mathrm{min}$ ), the signal was repeated in loop to cover the whole SPECT acquisition. 


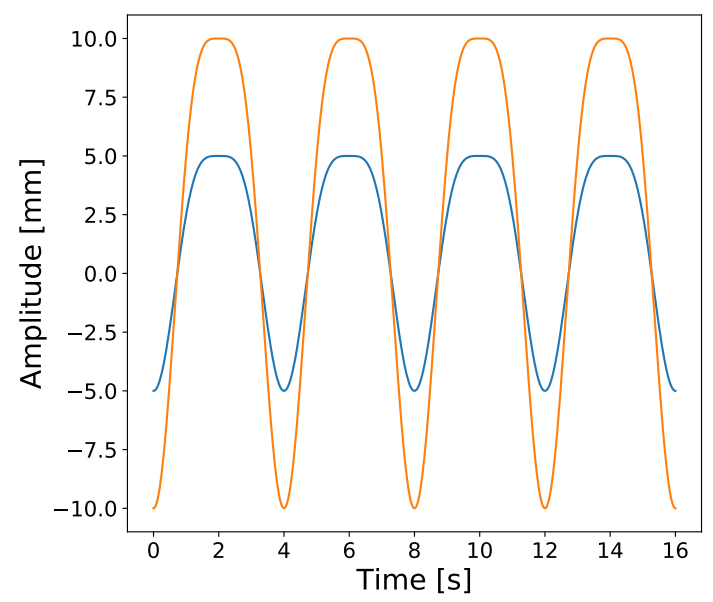

(a)

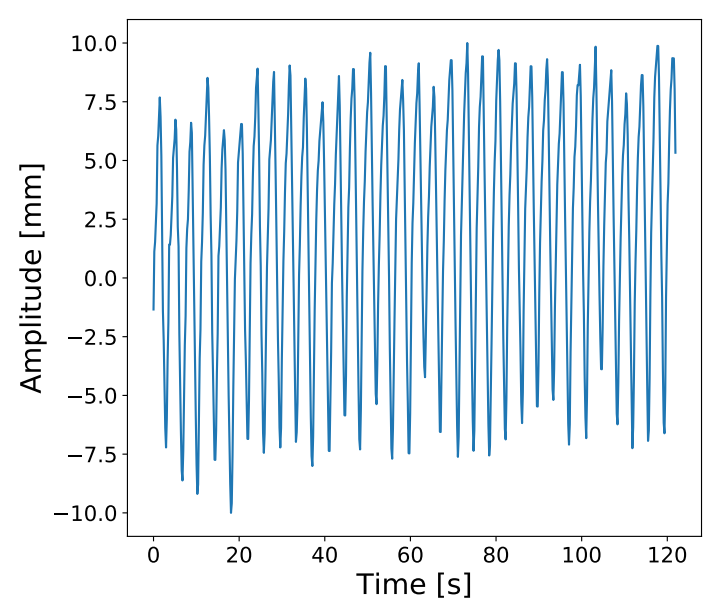

(b)

Fig. 1. Input signals for the phantom acquisitions. (a) $10 \mathrm{~mm}$ and $20 \mathrm{~mm}$ amplitude $\cos ^{4}$ function. (b) Patient-based signal.

Given the elapsed time between the first and the last acquisitions (70 $\mathrm{min})$ compared to the half-life of ${ }^{99 m} \mathrm{Tc}(6$ h), correction for radioactive decay was required. Therefore, a different correction factor was applied to each reconstructed image of the acquisitions with movement to account for the radioactive decay between acquisitions.

Patient acquisitions used for this study with written consent came from pre-treatment imaging for liver radioembolization. This step consists in injecting around $180 \mathrm{MBq}$ of ${ }^{99 m} \mathrm{Tc}$ macro aggregated albumin (MAA) inside the liver to assess lung shunt and extrahepatic uptake. Twenty distinct patients were included in this study.

\section{E. Quantitative analysis}

1) Breathing signal: Signal extraction was evaluated on the phantom and simulation data by comparing the phases of the extracted signals to the ones of the known input signals. The average period of the extracted signal was calculated and compared to the known period of the input signal.

For the phantom with the patient-based signal, the period was not known and not necessarily constant. Since the signal was played in loop during the acquisitions, the phase of the extracted signal should contain several repetitions of the phase of the reference signal. Therefore, we computed the phase of the reference signal and temporally registered it to the phase of the extracted signal to extract each repetition of the signal. Then, for each repeated signal, the temporal location of each end-inhale peak was compared against the one of the reference phase.

2) VOI delineation: A tumour volume of interest (VOI) was delineated on each 3D and 4D SPECT image. For the phantom acquisitions, the exact spherical volume containing the activity was known. In this case, a threshold was applied to the reconstructed SPECT images such that the volume of the VOI was the same as the one of the sphere. For the GATE simulation, the VOI of each frame of the 4D image was deduced from the known position of the tumor in the twenty phases used as input of the simulation. The VOI of the blurred 3D image was chosen to be the union of all 20 source positions.

On the patient SPECT images, the tumor contours were not available. In this case, a fixed threshold was used. The threshold value was set to $42 \%$ of the maximum value as is commonly used for SPECT image segmentation [37]. Following Kruis et al [38], the effective diameter (D) of the tumor was calculated to assess the tumor size:

$$
D=\sqrt[3]{\frac{6 V}{\pi}}
$$

with $V$ the volume of the VOI. The masks of the liver and the lungs were also retrieved. The segmentation was done on the CT images by the medical physicists of the cancer center using PLANET Dose (DOSIsoft, Cachan, France).

3) Tumor motion amplitude: We refer to the tumor motion amplitude as the Euclidean norm of the amplitude in the three directions of the coordinate system. The amplitude in one direction was measured on the gated 4D image as the difference between the maximum and the minimum positions of the VOI center of mass in that direction.

4) Activity recovery coefficient: The mean activity $\mathrm{A}$ in the VOI was measured for all reconstructed images to evaluate the effect of the method on the tumor activity, as well as its average $\bar{A}$ and its standard deviation in each set of lowcount 3D images. For the phantom acquisitions and the GATE simulation, the measured values for each frame of the 4D image and for the blurred 3D image were compared to the one in the $3 \mathrm{D}$ image without movement to obtain the relative difference

$$
\Delta \mathrm{A}=\frac{\mathrm{A}-\overline{\mathrm{A}}_{3 D}}{\overline{\mathrm{A}}_{3 D}} .
$$

For real patient acquisitions, it was not possible to have a $3 \mathrm{D}$ image without movement and the A values of the 4D image were compared to the average one of the blurred 3D image to evaluate the impact of the motion on the activity. We also computed the difference in diameters, $\Delta$ Diameter, to evaluate the influence of the 4D reconstruction on the effective diameter of the VOI.

As suggested by Park et al [39] and Kruis et al [38], the effect of respiratory motion in PET depends on the amplitude 
and the diameter of the tumor. We also investigated the correlation of $\Delta \mathrm{A}$ and $\Delta$ Diameter with the amplitude-to-diameter ratio and the amplitude alone for the patient acquisitions.

5) Lung shunt fraction: For the patient acquisitions, the lung shunt fraction (LSF) was computed for the blurred 3D image and each frame of the gated 4D image. The LSF is defined as follows:

$$
\mathrm{LSF}=\frac{\mathrm{TA}_{\text {lung }}}{\mathrm{TA}_{\text {liver }}+\mathrm{TA}_{\text {lung }}}
$$

where $\mathrm{TA}_{\text {lung }}$ and $\mathrm{TA}_{\text {liver }}$ are the sum of the activity inside the lung and the liver, respectively.

6) Tumor to normal liver ratio: The tumor to normal liver $(\mathrm{T} / \mathrm{N})$ ratio was also computed for the $3 \mathrm{D}$ and $4 \mathrm{D}$ images of the patient data:

$$
\mathrm{T} / \mathrm{N}=\frac{\mathrm{A}_{\text {tumor }}}{\mathrm{A}_{\text {liver }}}
$$

where $A_{\text {tumor }}$ is the mean activity in the tumor and $A_{\text {liver }}$ is the mean activity in the healthy liver.

\section{RESULTS}

\section{A. Signal extraction}

The Laplacian Eigenmaps algorithm extracted a signal close to the reference for the two phantom acquisitions with a regular respiratory signal and the simulation: the same average period (standard deviation) of $4.0(0.1) \mathrm{s}$ was found for the phantom and $4.0(0.2) \mathrm{s}$ for the simulation. After the registration of the phase of the patient-based signal to the phase of the extracted signal, 6 complete repetitions of the reference signal were found. The standard deviation of the time difference between corresponding end-inhale peaks was $0.1 \mathrm{~s}$. However, visual comparison of the reference and extracted signals for the first two camera stops of each phantom acquisition and the simulation (Fig. 2) indicates that the amplitude is not as accurately estimated as the phase which is computed from the end-inhale peaks.

\section{B. Simulation reconstruction}

An overview of the tumor at end-inhale and end-exhale positions in the sagittal plane is given in Fig. 3. The tumor deformation due to breathing motion can be seen on the 4D SPECT image. The measured amplitude of the tumor motion was $15.2 \mathrm{~mm}$ (10.4 $\mathrm{mm}$ in the superior-inferior direction, 10.4 $\mathrm{mm}$ in the antero-posterior direction and $3.8 \mathrm{~mm}$ in the leftright direction).

The mean activity in the VOI of the blurred 3D image was on average $22.8 \%$ below the ones of the images without movement (Fig. 4). The average loss of activity for the 4D image was only $-2.6 \%$, meaning that we recovered $20.2 \%$ more activity than the uncorrected reconstruction.

\section{Phantom reconstructions}

One slice of the 3D image along with the end-inhale, midexhale and end-exhale positions of the 4D image is shown in Fig. 5 for the three acquisitions of the dynamic thorax phantom. The blur around the tumor in the $3 \mathrm{D}$ images is reduced in the 4D images and the tumor motion can be appreciated visually despite the noise increase.

The axial position of the VOI center of mass is given in Fig. 6. The movement amplitude of the center of mass is 18.7 $\mathrm{mm}$ for the $20 \mathrm{~mm}$ amplitude signal (Fig. 6a), $9.1 \mathrm{~mm}$ for the $10 \mathrm{~mm}$ amplitude signal (Fig. 6b) and $12.7 \mathrm{~mm}$ for the patient-based signal. Furthermore, the change of position of the center of mass in the 4D image matches the input $\cos ^{4}$ function (Fig. 6a and 6b).

The change of activity $\mathrm{A}$ for the phantom acquisitions are shown in Fig. 7. The mean value inside the VOI on the 3D uncorrected image was $19.4 \%$ below the reference for the $20 \mathrm{~mm}$ amplitude signal, whereas the activity loss was $12.8 \%$ and $7.5 \%$ for the patient and $10 \mathrm{~mm}$ amplitude signal, respectively. The 4D reconstruction yielded activities closer to the reference: $-2.4 \%,-2.8 \%$ and $-4.5 \%$ on average for the $20 \mathrm{~mm}, 10 \mathrm{~mm}$, and patient-based signal, respectively. The standard deviation of $\mathrm{A}$ along the 8 low-count 3D images of the reference was $0.6 \%$. The standard deviations of the blurred $3 \mathrm{D}$ images were $0.7 \%, 1.6 \%$ and $0.8 \%$ for the $20 \mathrm{~mm}, 10 \mathrm{~mm}$ and patient-based signal, respectively. These differences are unexpected but are not significant $(p>0.2$, Levene test for equal variances) because they are calculated from 8 samples only.

\section{Patient reconstructions}

For the 20 patient acquisitions, a total of 29 tumors were delineated and included in the study: one tumor per acquisition for 15 acquisitions, two tumors for two acquisitions, three tumors for two acquisitions, and four tumors for one acquisition. In all cases, the Laplacian Eigenmaps algorithm provided a respiratory signal which seemed appropriate for phase gating. The average tumor amplitude was $8.1 \mathrm{~mm}$ and was generally difficult to appreciate visually on the reconstructed images with $4.4 \mathrm{~mm}$ voxel size. The largest tumor motion was 16.4 $\mathrm{mm}$ and is shown in Fig. 8. The extracted signals for the two patients with the largest tumor motion along with the change of center of mass in the 4D image are given in Fig. 9. It can be observed that the biggest contribution to the tumor motion is in the superior-inferior direction in both cases (Fig. 9c and 9d).

The average value of $\Delta \mathrm{A}$ over the 8 -frames 4D SPECT images $(\overline{\Delta \mathrm{A}})$ was between $-0.1 \%$ and $11.9 \%$ with an average value of $3.2 \%$, meaning that higher activity was recovered compared to the 3D SPECT images. The standard deviation of $\Delta \mathrm{A}$ for the 8 frames of one 4D reconstruction was on average $3.5 \%$ (between $1.11 \%$ and $8.9 \%$ ), which denotes some variability in the recovered activity from one frame to the other. Compared to the uncorrected 3D reconstruction, the size of the VOI decreased on average by $-3.8 \%$ (between $1.72 \%$ and $-18.4 \%$ ) in the $4 \mathrm{D}$ reconstruction.

There seems to be a correlation between the tumor motion amplitude and the change in activity $\overline{\Delta \mathrm{A}}$ (Fig. 10a) or the change in diameter $\Delta$ Diameter (Fig. 10b). The correlation with the amplitude-to-diameter ratio was better (Fig. 10c and 10d), with a trend suggesting that the change in activity increases with the ratio and that the change in diameter decreases with the ratio. 


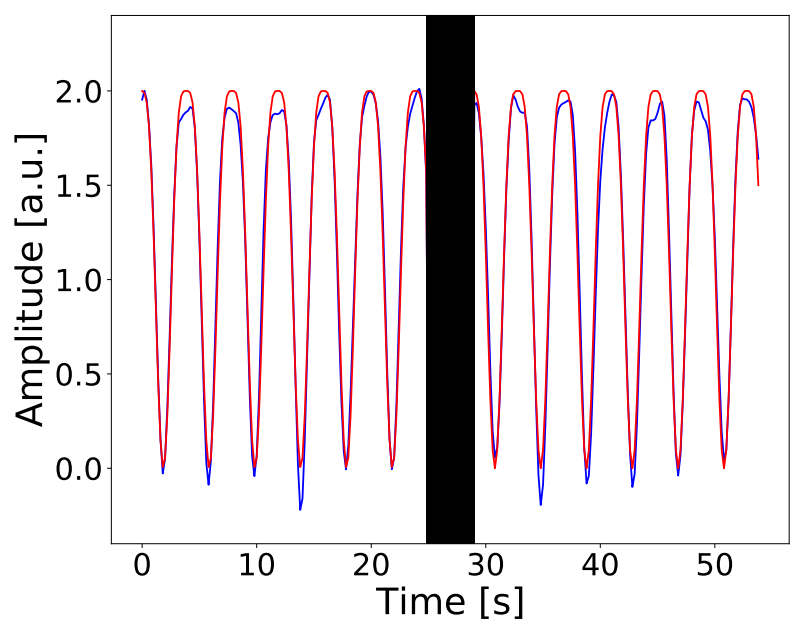

(a) $20 \mathrm{~mm}$ amplitude signal

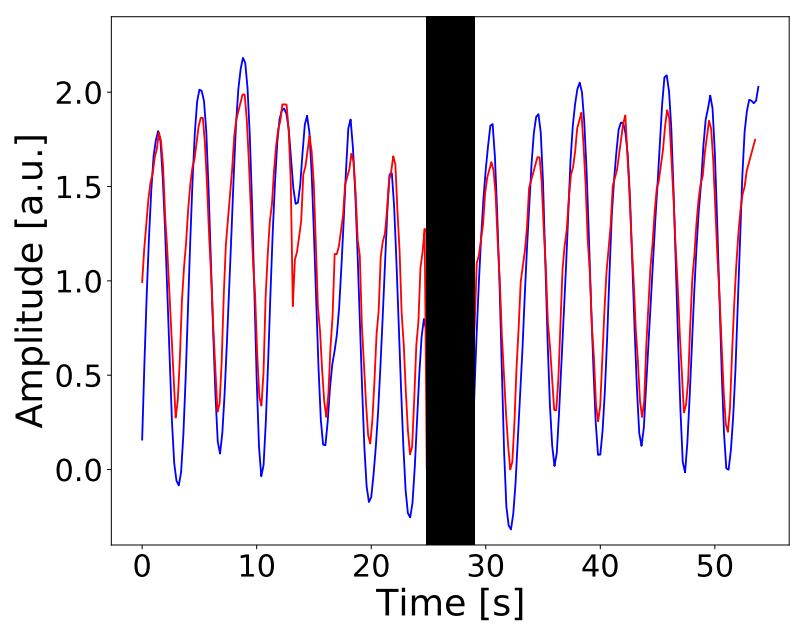

(c) Patient-based signal

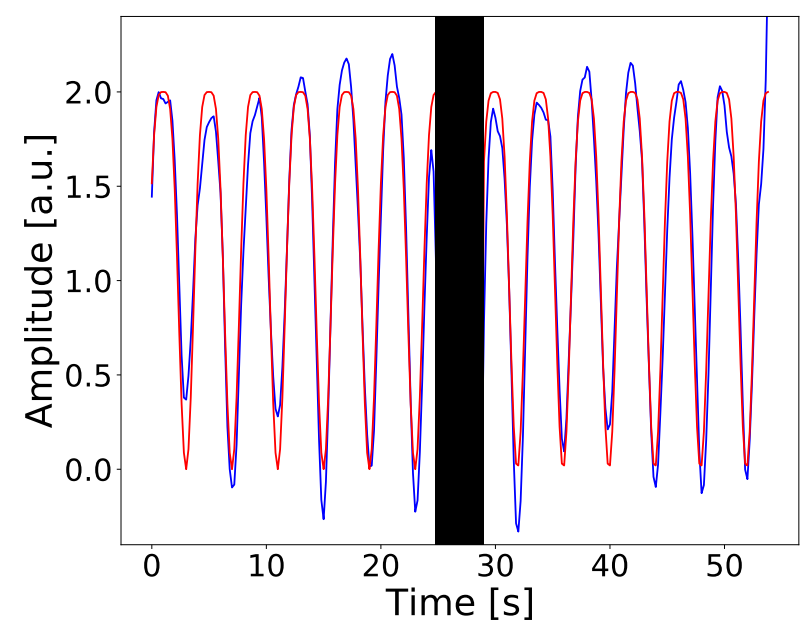

(b) $10 \mathrm{~mm}$ amplitude signal

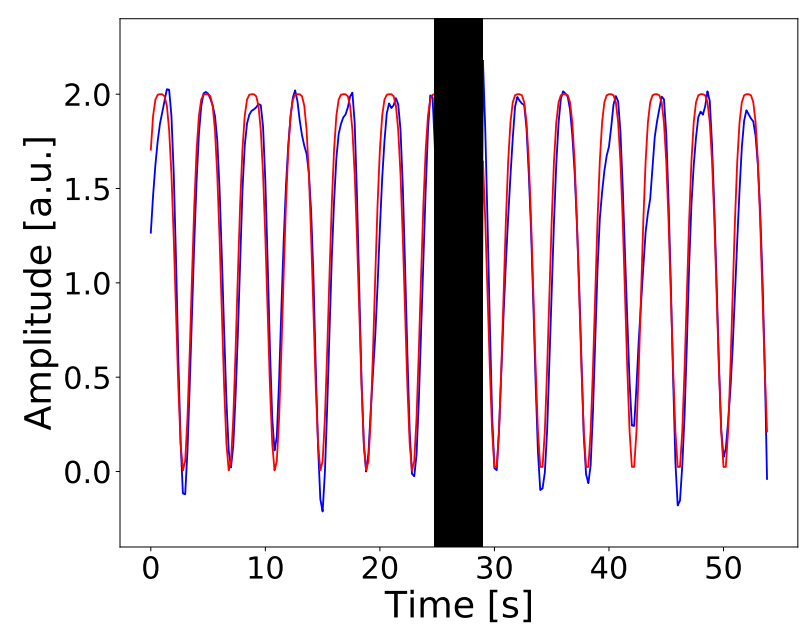

(d) Simulation

Fig. 2. Extracted signal for the three phantom acquisitions and the simulation compared to the reference signal. The signals are shown for the first two camera stops. The black rectangle corresponds to the time between two camera stops.

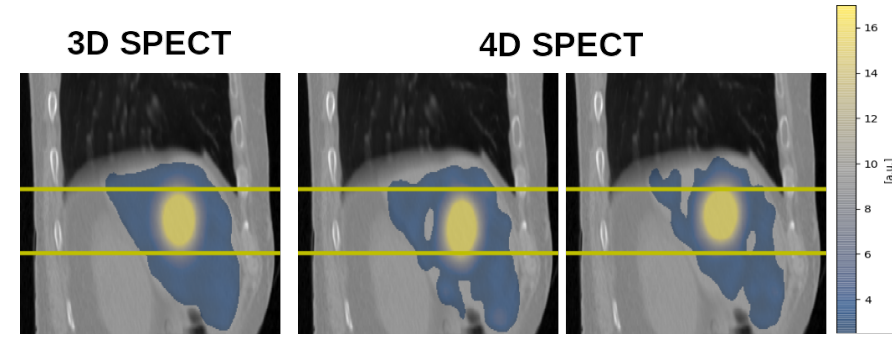

Fig. 3. Reconstructed SPECT images of the GATE simulation. From left to right: blurred 3D SPECT, frame 1 of the 4D image (end-inhale), frame 5 (end-exhale).

The relative difference between the LSF of the blurred 3D images and each frame of the $4 \mathrm{D}$ images $(\triangle \mathrm{LSF})$ is shown in Fig. 11. The average value of $\Delta \mathrm{LSF}$ over the 8 -frames $4 \mathrm{D}$ images $(\overline{\Delta \mathrm{LSF}})$ was between $-1.0 \%$ and $2.3 \%$ with an average value of $0.6 \%$. High variability can be observed between frames of the 4D images with an average standard deviation of $16.6 \%$ (between $2.7 \%$ and $42.2 \%$ ).

Similarly, the $\mathrm{T} / \mathrm{N}$ ratios of the blurred $3 \mathrm{D}$ images were compared to the ones of the 4D images (Fig. 12). The average relative difference $\overline{\Delta \mathrm{T} / \mathrm{N}}$ was on average $3.2 \%$ (between $3.6 \%$ and $9.7 \%$ ) and the average standard deviation of $\Delta \mathrm{T} / \mathrm{N}$ over the frames was $3.6 \%$ (between $1.3 \%$ and $8.5 \%$ ).

\section{DISCUSSION}

The proposed respiration-gated 4D reconstruction was applied to simulated, phantom and patient SPECT acquisitions. The breathing signal was directly extracted from the acquired 


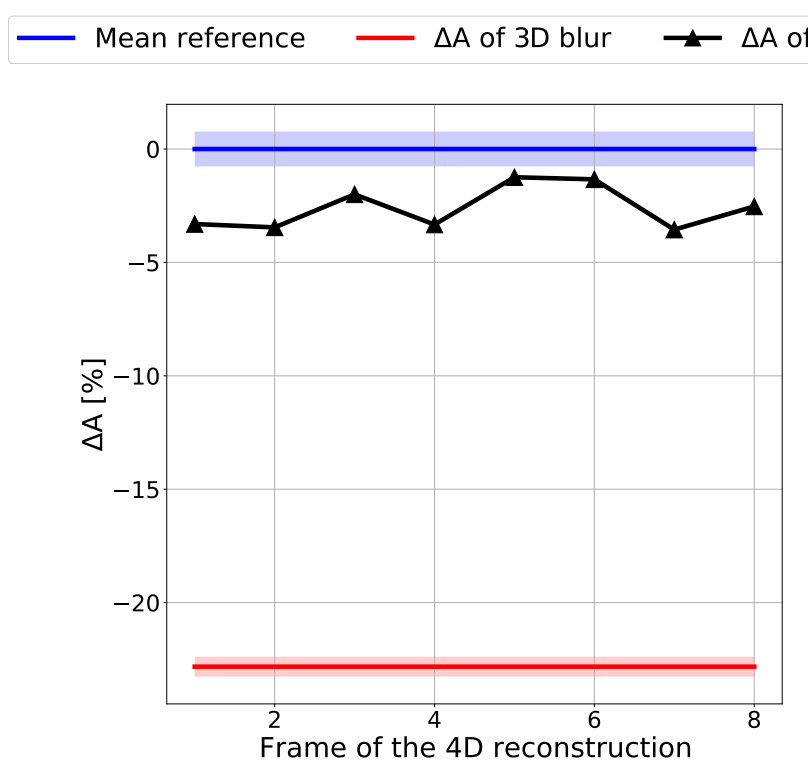

Fig. 4. Differences in mean activity recovery inside the tumor for the GATE simulation. The color-filled areas correspond to plus/minus one standard deviation of the mean activity inside the tumor of the set of the $83 \mathrm{D}$ images.
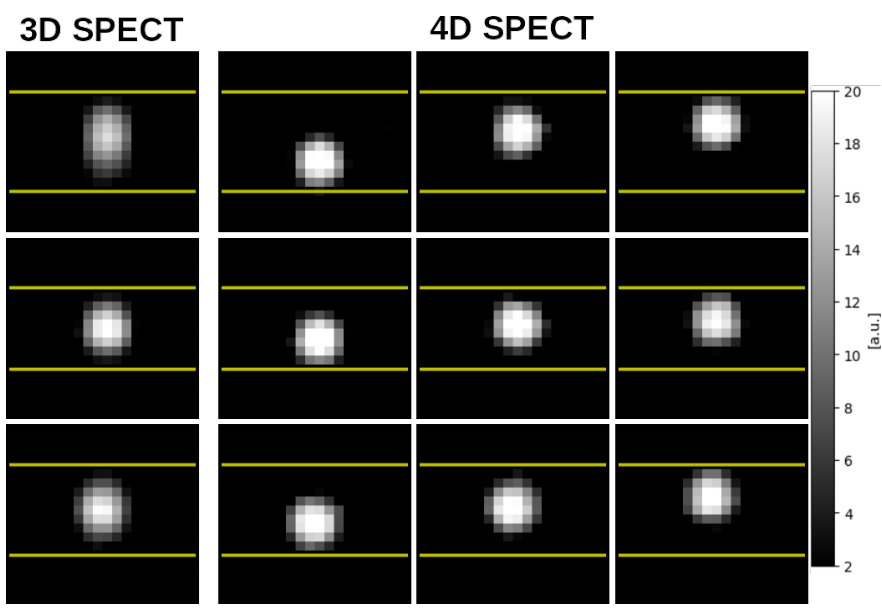

Fig. 5. Reconstructed SPECT images of the dynamic phantom. Top: $\cos ^{4}$ signal with $20 \mathrm{~mm}$ amplitude. Middle: $\cos ^{4}$ signal with $10 \mathrm{~mm}$ amplitude. Bottom: patient signal. From left to right: blurred 3D image, frame 1 of the gated 4D image (end-inhale), frame 3 (mid-exhale), frame 5 (end-exhale).

data without any additional hardware. Therefore, the proposed method is compatible with clinical constraints of any SPECT when list-mode data are available.

The ability of the Laplacian algorithm to extract a useful respiratory signal for gating was evaluated with the dynamic phantom acquisitions and the simulation. The positions of the peaks of the extracted signals matched well the ones of the references. For the phantom acquisitions, the activity was only located inside the tumor without background activity which might facilitate the signal extraction. For patients treated with radioembolization, a large amount of the injected activity is also located in the tumor and the phantom seems reasonable. Moreover, the signal extracted from the simulation, in which background activity was present in the liver, was also well correlated with the reference. The performance of the algorithm

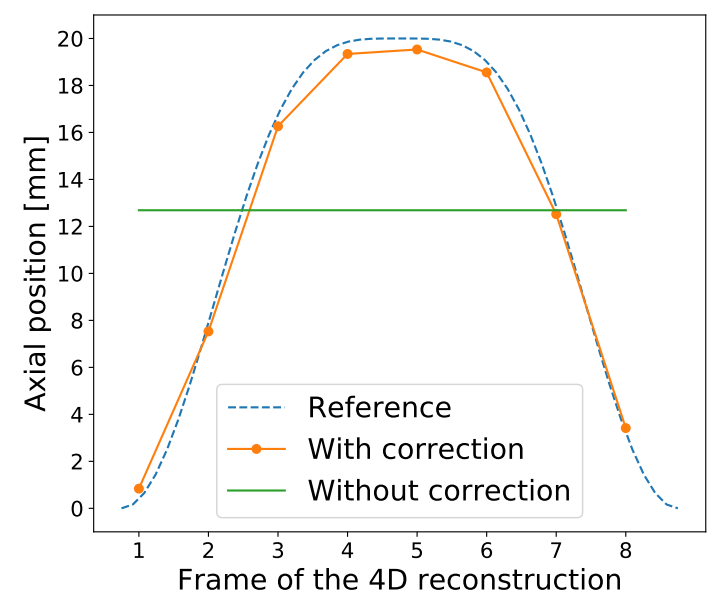

(a) $20 \mathrm{~mm}$ amplitude signal

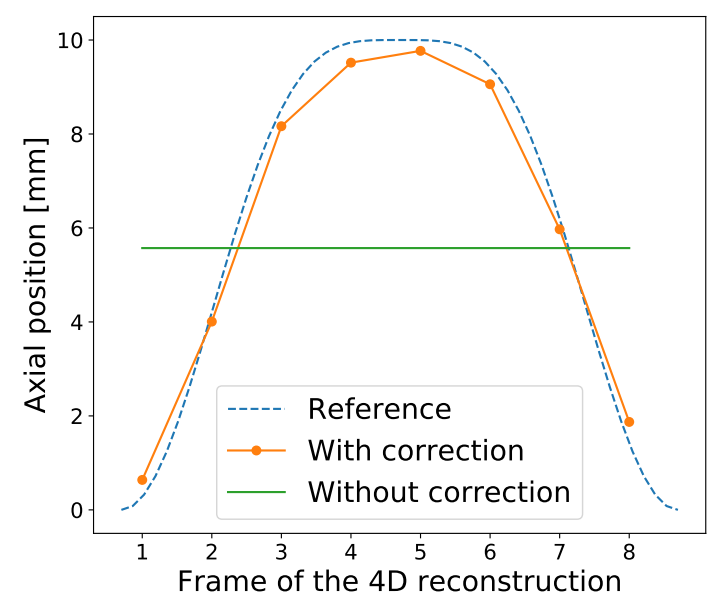

(b) $10 \mathrm{~mm}$ amplitude signal

Fig. 6. Axial position of the center of mass inside the VOI of the dynamic phantom SPECT images. Dotted line represent the input $\cos ^{4}$ function.

might be challenged by more diffuse activity.

In cardiac SPECT imaging, some studies have shown that amplitude binning outperforms phase binning [9], [10]. However, in a first approach, we used phase binning to reconstruct the $4 \mathrm{D}$ images as is commonly done clinically in both 4D CT [28], [40] and 4D PET/CT [39], [41] scan. Assessing the quality of amplitude binning with this data-driven approach was out of the scope of this work but might be a promising way to improve the method.

For the experimental datasets, the 4D reconstruction allowed the measure of higher activity compared to the blurred 3D reconstruction: $17 \%, 8.3 \%$ and $5.1 \%$ more activity for the three phantom acquisitions and $20.2 \%$ for the GATE simulation. Therefore, the $\overline{\mathrm{A}}$ value in the $4 \mathrm{D}$ images were significantly closer to the reference $3 \mathrm{D}$ reconstruction without breathing motion: between $-2.7 \%$ and $5.1 \%$ for the three phantom acquisitions and $-1.0 \%$ for the simulation.

In this study, the pre-treatment 4D SPECT images of liver patients treated with radioembolization showed an average $3.2 \%$ increase (up to $11.9 \%$ ) of the mean activity value inside the tumor compared to the $3 \mathrm{D}$ images. This activity recovery 


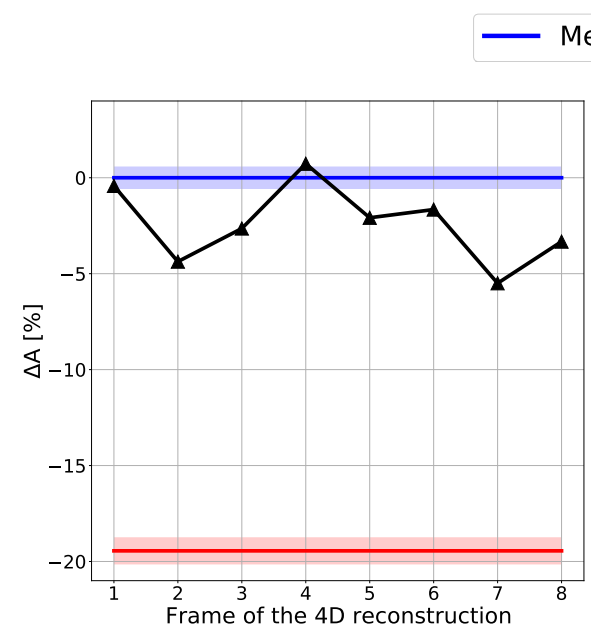

(a) $20 \mathrm{~mm}$ amplitude signal

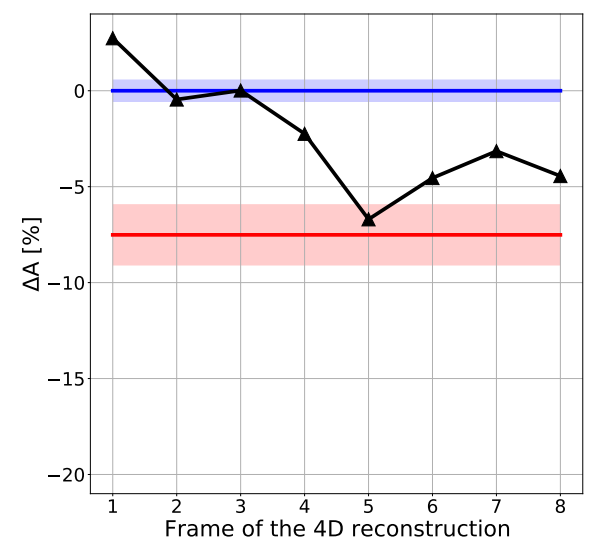

(b) $10 \mathrm{~mm}$ amplitude signal

Fig. 7. Differences of mean activity inside the VOI for the phantom reconstructions. The color-filled areas correspond to the standard deviations of the mean activity inside the tumor of the set of the $83 \mathrm{D}$ images.
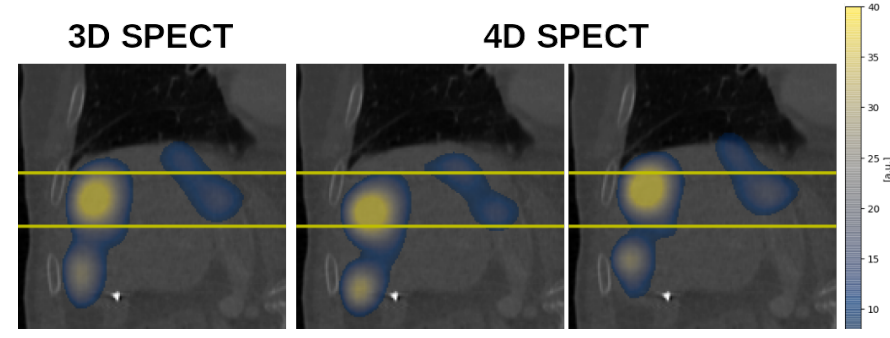

Fig. 8. Reconstructed SPECT images of one patient. From left to right: blurred 3D image, frame 1 of the gated 4D image (end-inhale), frame 5 (end-exhale).

was negative for only one tumor with a value of $-0.1 \%$. We also noted a $3.8 \%$ average decrease of the size of the VOI with an $18.4 \%$ maximum.

Following the work of Kruis et al on breathing motion in PET [38], we have also investigated the correlation of the mean activity inside the VOI with the amplitude of the motion and the amplitude-to-diameter ratio. The increase of $\overline{\Delta \mathrm{A}}$ was better correlated with the increase of the amplitude-to-diameter ratio than with the amplitude alone. It suggests that the proposed method is more relevant for small tumors and/or large motion.

The mean activity was variable between frames of the 4D images. For the patient images, the uncertainty was partly due to the method used for the VOI selection. Indeed, the choice of the VOI relies on the maximum value of the reconstructed SPECT image which might suffer from noise. The noise was found significant because each frame of the 4D SPECT image uses 8 times less data than the clinical 3D image reconstructed from the same acquisition. It could also explain why the activity A was above the reference in some frames of the phantom and simulation 4D images.

Noise could be reduced by increasing the injected activity to increase the amount of events for reconstructing each frame of the 4D SPECT image. Another approach is to use advanced reconstruction techniques like motion-compensated reconstruction [38], [42], [43] that allow reconstruction of a corrected 3D image with all the available data.
The mean difference of LSF between the 3D and 4D reconstructions was small: $0.6 \%$ on average. However, the variation of LSF between frames was important. This indicates that the respiratory motion correction has an effect on the LSF computation. For the $\mathrm{T} / \mathrm{N}$ ratio, the value in the $4 \mathrm{D}$ gated images was higher by $3.2 \%$ on average compared to the blurred 3D images, but also with large variability between frames. The average ratio was negative $(-3.6 \%)$ for one tumor only.

The variability of the $\mathrm{LSF}$ and the $\mathrm{T} / \mathrm{N}$ ratio over the frames of the 4D image may be due to the use of a $3 \mathrm{D}$ free breathing CT to correct for attenuation correction and to segment the lungs and the liver. Indeed, Lu et al [44] showed on simulated 3D ${ }^{99 m}$ Tc-MAA SPECT/CT that the choice of the attenuation map affects the computation of the $\mathrm{LSF}$ and $\mathrm{T} / \mathrm{N}$ ratio. The best results were obtained with a mid-respiration CT and end-expiration CT appeared to be the second best choice. In this study, only free breathing CT was available which is the image of a random state of the respiration. Therefore, the computations of the LSF and the $\mathrm{T} / \mathrm{N}$ ratio were subject to over or under estimation depending of the frame of the $4 \mathrm{D}$ reconstructions, which could explain the observed variability. Moreover, it has been shown that mismatches between the emission and transmission scans for attenuation correction cause artifacts and can introduce errors in measured activitied on both PET [45], [46] and cardiac SPECT images [47], [48]. This may also be an explanation of the variability of the A value observed in the 4D images. Indeed, the highest standard deviation of $\Delta \mathrm{A}$ in the 8 frames of one 4D image was measured for a tumor located at the border between the liver and the lung. In the fifth frame of the 4D SPECT image, corresponding to the end-exhale position, a part of the tumor matched the lung position in the attenuation map. Consequently, the A values for the fourth and fifth frames was almost $25 \%$ smaller than the ones in the first and eighth frames corresponding to end-inhale. Similar observations were made for three other tumors. Several solutions have been investigated to improve attenuation correction of $4 \mathrm{D}$ SPECT and PET 


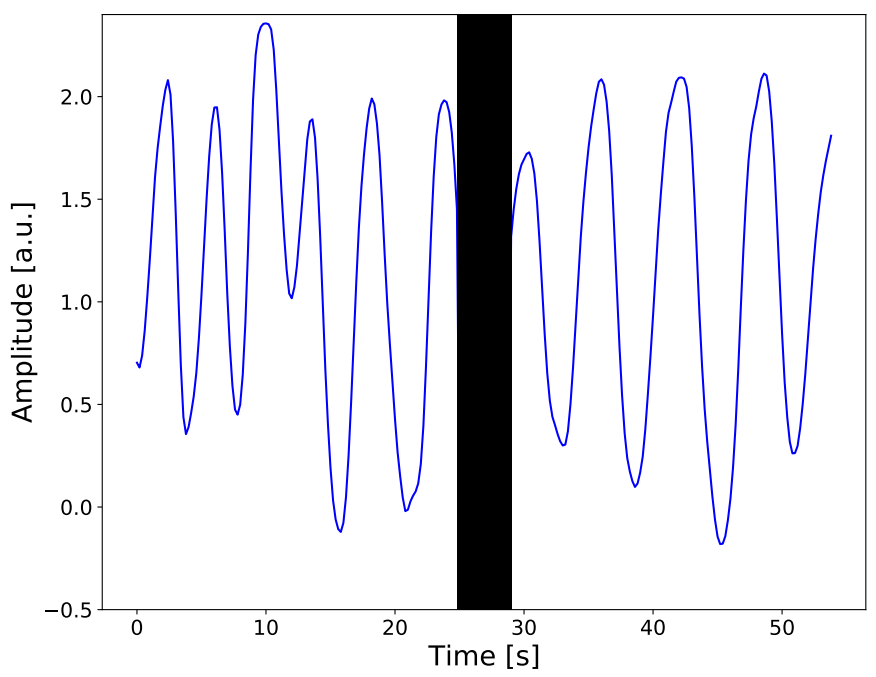

(a) Patient 15

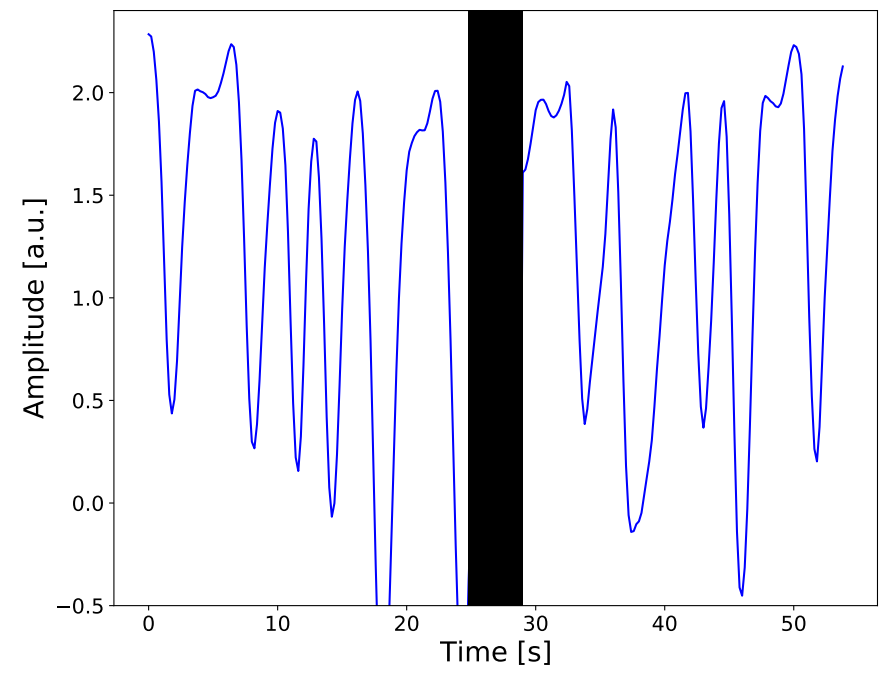

(b) Patient 16

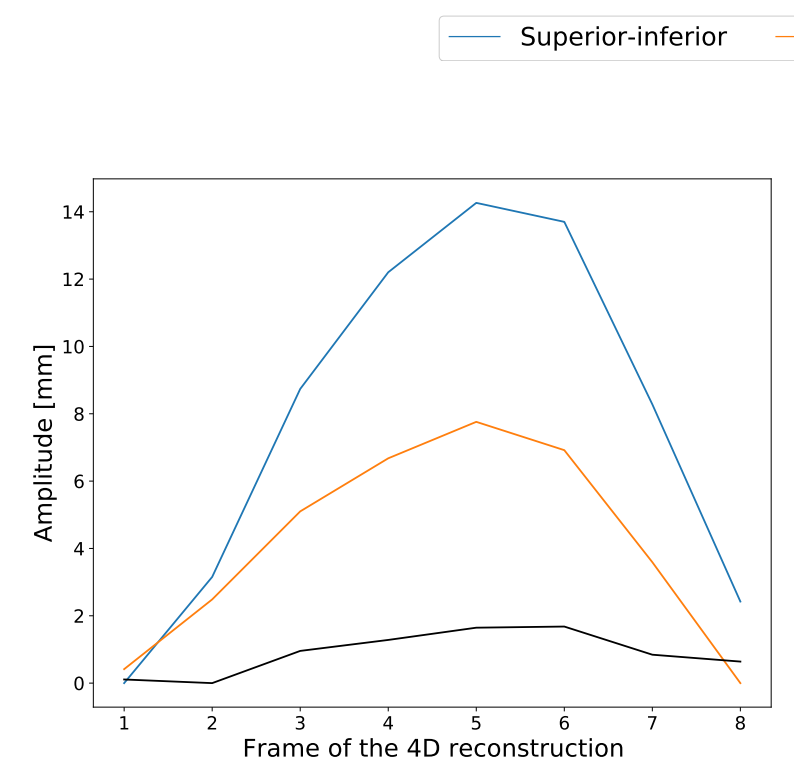

(c) Patient 15

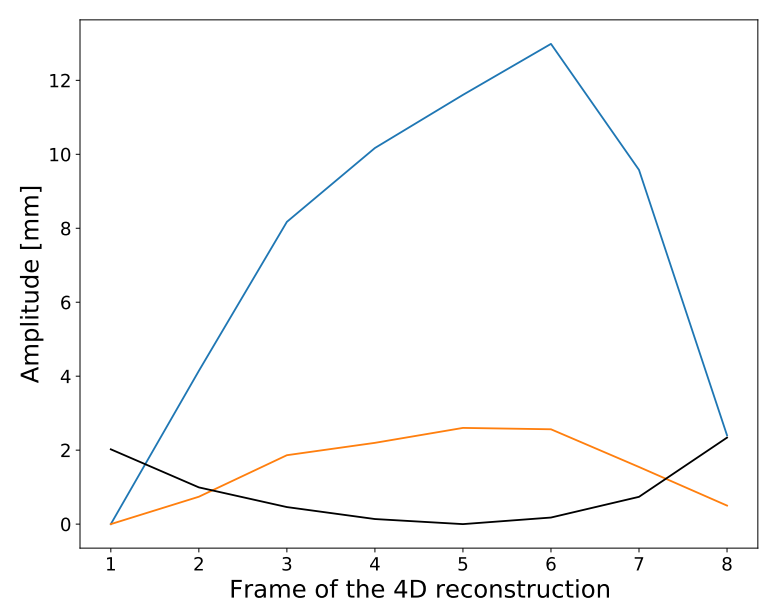

(d) Patient 16

Fig. 9. Extracted signal of the first two camera stops for the two patients with the largest tumor motion (a,b) and the motion of the center of mass in the corresponding 4D images (c, d).

images, e.g., using 4D CT [49], [50] or machine learning approaches [51], which were beyond the scope of this study.

The proposed method was applied to liver tumors only but it should also be applicable to other sites, e.g., lung tumors and other upper-abdominal tumors which are moving with breathing provided that the signal extraction still is efficient. It could also be used for other types of SPECT images such as ${ }^{177} \mathrm{Lu}$-based treatments.

\section{CONCLUSION}

We have developed and evaluated a fully data-driven respiration-gated SPECT reconstruction applied to liver radioembolization. The breathing signal was extracted from the list-mode data using the Laplacian Eigenmaps algorithm. Breathing motion correction enables activity recovery and higher T/N ratio compared to conventional 3D SPECT. Frameto-frame LSF variability also indicates the need to account for motion in its quantification.

\section{ACKNOWLEDGMENT}

This work was performed within the framework of the SIRIC LYriCAN Grant INCa-INSERM-DGOS-12563, the LABEX PRIMES (ANR-11-LABX-0063) of Université de Lyon, within the program Investissements d'Avenir (ANR11-IDEX-0007) operated by the ANR, the NADIAM PackAmbition Rhône-Alpes region project and the POPEYE ERA PerMed 2019 project (ANR-19-PERM-0007-04).

\section{REFERENCES}

[1] R. Bastiaannet, M. A. Viergever, and H. W. A. M. de Jong, "Impact of respiratory motion and acquisition settings on SPECT liver dosimetry 


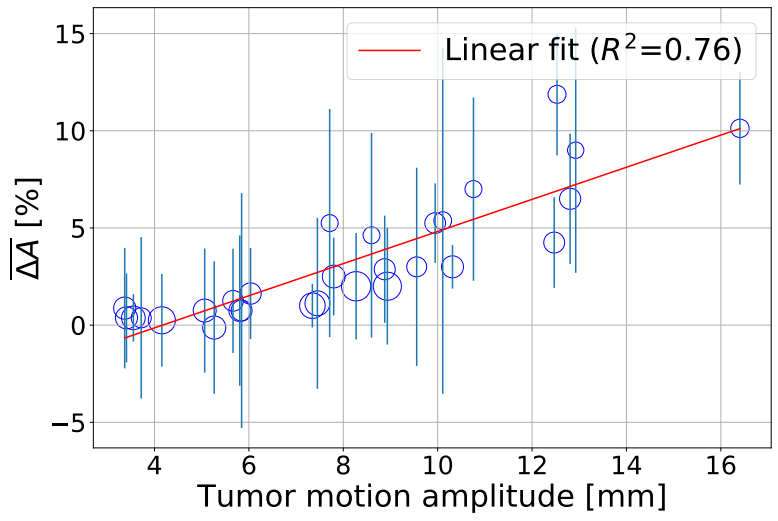

(a)

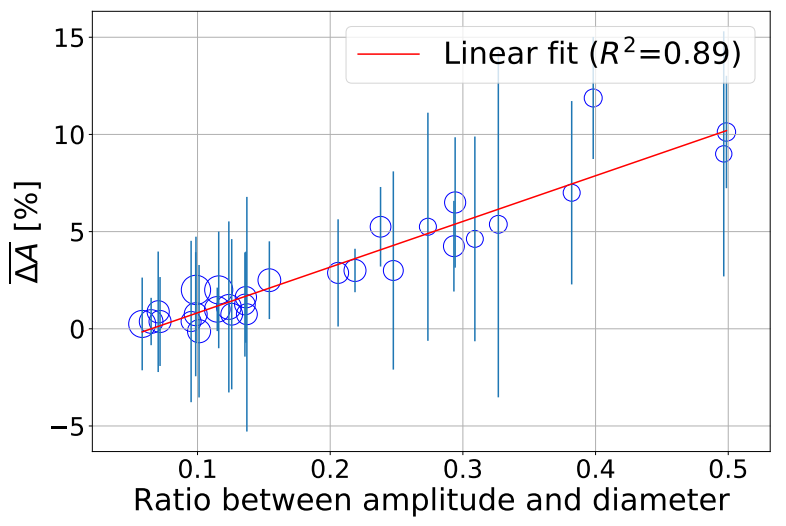

(c)

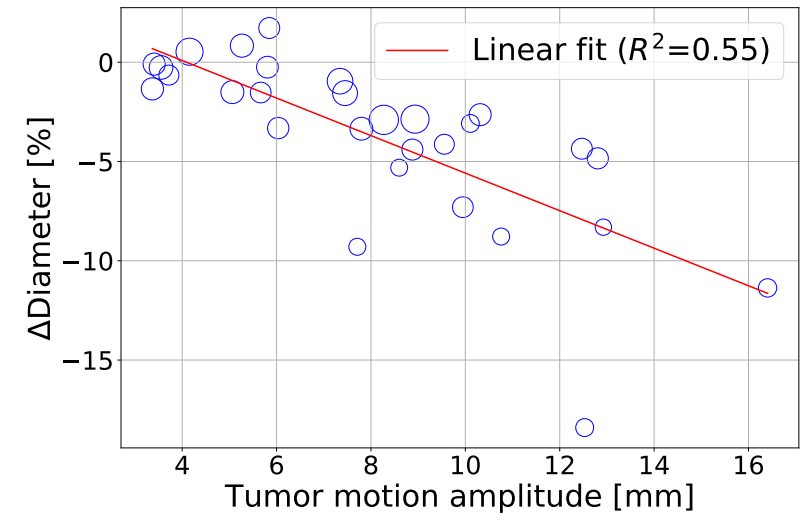

(b)

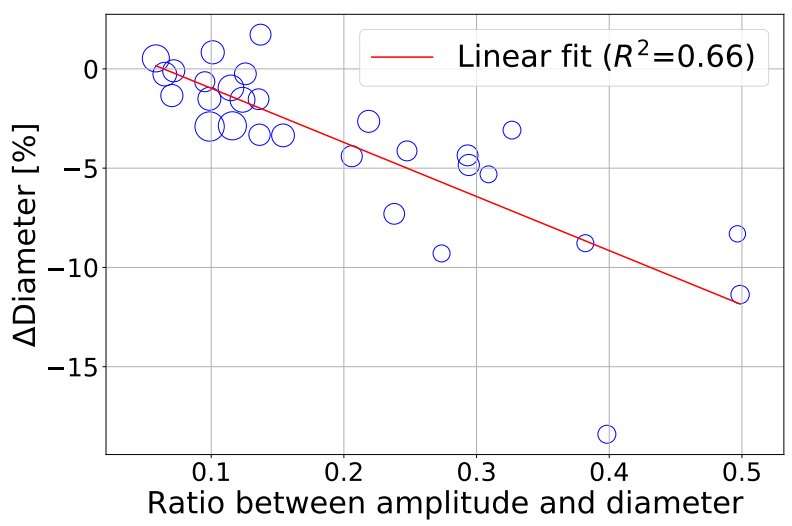

(d)

Fig. 10. Differences in mean activity recovery $\Delta \overline{\mathrm{A}}$ and diameter of the VOI $\Delta$ Diameter as a function of the tumor motion amplitude (a-b), and to the amplitude-to-diameter ratio (c-d). The error bars correspond to the standard deviation of the $\Delta \mathrm{A}$ values in the 8 frames of the $4 \mathrm{D}$ reconstruction. The size of the points is proportional to the size of the VOIs. The slope of the four linear regressions is significantly different from $0(p<0.01)$.

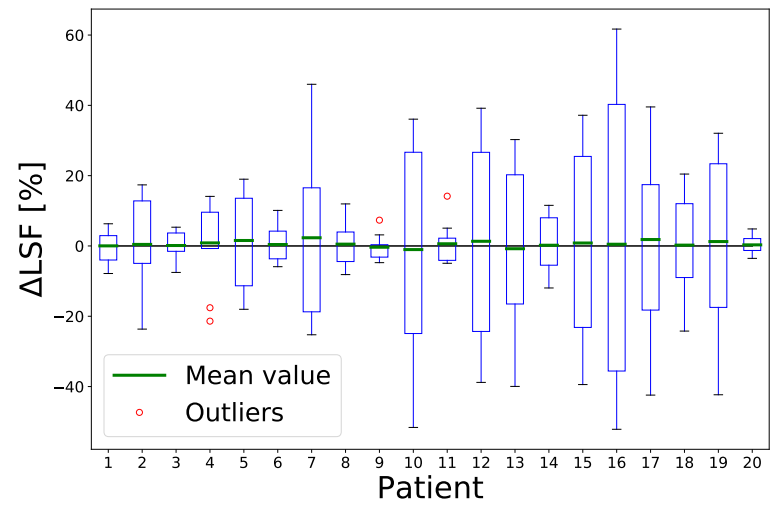

Fig. 11. Boxplot of the relative difference between the LSF of the blurred 3D images and each frame of the 4D images. The box represents the interquartile range (IQR), i.e., the values between the 25 th percentile (lower quartile) and the 75th percentile (upper quartile). The end of the whiskers represent the minimum and maximum value excluding outliers. A value is considered as an outlier when it is outside $1.5 * \mathrm{IQR}$ below the lower quartile or above the upper quartile.

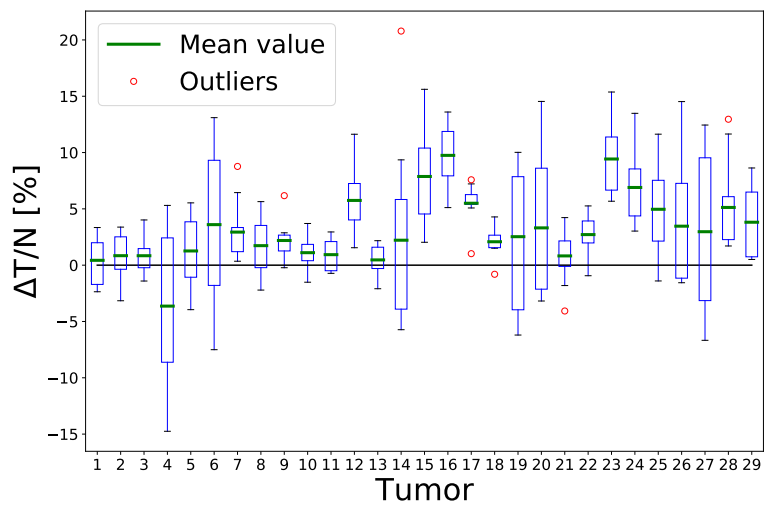

Fig. 12. Boxplot of the relative difference between the $\mathrm{T} / \mathrm{N}$ ratio of the blurred 3D images and each frame of the 4D images. See the caption of Fig. 11 for the signification of the boxes and their whiskers.

for radioembolization," Medical Physics, vol. 44, no. 10, pp. 5270-5279, Oct. 2017.

[2] R. L. Eisner, T. Noever, D. Nowak, W. Carlson, D. Dunn, J. Oates, K. Cloninger, H. A. Liberman, and R. E. Patterson, "Use of cross- 
correlation function to detect patient motion during SPECT imaging," Journal of Nuclear Medicine: Official Publication, Society of Nuclear Medicine, vol. 28, no. 1, pp. 97-101, Jan. 1987.

[3] R. R. Fulton, B. F. Hutton, M. Braun, B. Ardekani, and R. Larkin, "Use of 3D reconstruction to correct for patient motion in SPECT," Physics in Medicine and Biology, vol. 39, no. 3, pp. 563-574, Mar. 1994.

[4] W. J. Geckle, T. L. Frank, J. M. Links, and L. C. Becker, "Correction for patient and organ movement in SPECT: Application to exercise thallium201 cardiac imaging," Journal of Nuclear Medicine, vol. 29, no. 4, pp. 441-450, Jan. 1988.

[5] T. L. Faber, C. D. Cooke, R. D. Folks, J. P. Vansant, K. J. Nichols, E. G DePuey, R. I. Pettigrew, and E. V. Garcia, "Left ventricular function and perfusion from gated SPECT perfusion images: An integrated method," Journal of Nuclear Medicine: Official Publication, Society of Nuclear Medicine, vol. 40, no. 4, pp. 650-659, Apr. 1999.

[6] F. Mannting and M. G. Morgan-Mannting, "Gated SPECT with Technetium-99m-Sestamibi for Assessment of Myocardial Perfusion Abnormalities," Journal of Nuclear Medicine, vol. 34, no. 4, pp. 601608, Apr. 1993.

[7] G. Kovalski, O. Israel, Z. Keidar, A. Frenkel, J. Sachs, and H. Azhari, "Correction of heart motion due to respiration in clinical myocardial perfusion SPECT scans using respiratory gating," Journal of Nuclear Medicine: Official Publication, Society of Nuclear Medicine, vol. 48, no. 4, pp. 630-636, Apr. 2007.

[8] C. Chan, M. Harris, M. Le, J. Biondi, Y. Grobshtein, Y.-H. Liu, A. J. Sinusas, and C. Liu, "End-expiration respiratory gating for a highresolution stationary cardiac SPECT system," Physics in Medicine and Biology, vol. 59, no. 20, pp. 6267-6287, Sep. 2014.

[9] D. Zhang, P. H. Pretorius, M. Ghaly, Q. Zhang, M. A. King, and G. S. P. Mok, "Evaluation of different respiratory gating schemes for cardiac SPECT," Journal of Nuclear Cardiology, vol. 27, no. 2, pp. 634-647, Apr. 2020.

[10] D. Zhang, J. Sun, P. H. Pretorius, M. King, and G. S. P. Mok, "Clinical evaluation of three respiratory gating schemes for different respiratory patterns on cardiac SPECT," Medical Physics, vol. 47, no. 9, pp. 42234232, Sep. 2020.

[11] R. D. Beach, P. H. Pretorius, G. Boening, P. P. Bruyant, B. Feng, R. R. Fulton, M. A. Gennert, S. Nadella, and M. A. King, "Feasibility of Stereo-Infrared Tracking to Monitor Patient Motion During Cardiac SPECT Imaging," IEEE transactions on nuclear science, vol. 51, no. 5 II, pp. 2693-2698, Oct. 2004

[12] X. A. Li, C. Stepaniak, and E. Gore, "Technical and dosimetric aspects of respiratory gating using a pressure-sensor motion monitoring system," Medical Physics, vol. 33, no. 1, pp. 145-154, 2006.

[13] T. Zhang, H. Keller, M. J. O’Brien, T. R. Mackie, and B. Paliwal, "Application of the spirometer in respiratory gated radiotherapy," Medical Physics, vol. 30, no. 12, pp. 3165-3171, 2003.

[14] R. I. Berbeco, S. Nishioka, H. Shirato, G. T. Y. Chen, and S. B. Jiang, "Residual motion of lung tumours in gated radiotherapy with external respiratory surrogates," Physics in Medicine and Biology, vol. 50, no. 16, pp. 3655-3667, Aug. 2005.

[15] D. P. Gierga, J. Brewer, G. C. Sharp, M. Betke, C. G. Willett, and G. T. Y. Chen, "The correlation between internal and external markers for abdominal tumors: Implications for respiratory gating," International Journal of Radiation Oncology, Biology, Physics, vol. 61, no. 5, pp. 1551-1558, Apr. 2005.

[16] P. P. Bruyant, M. A. King, and P. H. Pretorius, "Correction of the respiratory motion of the heart by tracking of the center of mass of thresholded projections: A simulation study using the dynamic MCAT phantom," IEEE Transactions on Nuclear Science, vol. 49, no. 5, pp. 2159-2166, Oct. 2002.

[17] A. L. Kesner and C. Kuntner, "A new fast and fully automated software based algorithm for extracting respiratory signal from raw PET data and its comparison to other methods: Automated data based respiratory signal acquisition," Medical Physics, vol. 37, no. 10, pp. 5550-5559, Sep. 2010.

[18] J. C. Sanders, P. Ritt, T. Kuwert, A. H. Vija, and A. K. Maier, "Fully Automated Data-Driven Respiratory Signal Extraction From SPECT Images Using Laplacian Eigenmaps," IEEE transactions on medical imaging, vol. 35, no. 11, pp. 2425-2435, Nov. 2016.

[19] K. Thielemans, S. Rathore, F. Engbrant, and P. Razifar, "Deviceless gating for PET/CT using PCA," Nuclear Science Symposium and Medical Imaging Conference (NSS/MIC), Oct. 2011.

[20] D. Daou, R. Sabbah, C. Coaguila, and H. Boulahdour, "Feasibility of data-driven cardiac respiratory motion correction of myocardial perfusion CZT SPECT: A pilot study," Journal of Nuclear Cardiology:
Official Publication of the American Society of Nuclear Cardiology, vol. 24, no. 5, pp. 1598-1607, Oct. 2017.

[21] Y. Kitamura, S. Baba, T. Isoda, Y. Maruoka, S. Kawanami, K. Himuro, M. Sasaki, and H. Honda, "The Efficiency of Respiratory-gated 18FFDG PET/CT in Lung Adenocarcinoma: Amplitude-gating Versus Phase-gating Methods," Asia Oceania Journal of Nuclear Medicine and Biology, vol. 5, no. 1, pp. 30-36, 2017.

[22] L. Le Meunier, R. Maass-Moreno, J. A. Carrasquillo, W. Dieckmann, and S. L. Bacharach, "PET/CT imaging: Effect of respiratory motion on apparent myocardial uptake," Journal of Nuclear Cardiology: Official Publication of the American Society of Nuclear Cardiology, vol. 13, no. 6, pp. 821-830, Nov. 2006.

[23] C. Liu, L. A. Pierce, A. M. Alessio, and P. E. Kinahan, "The impact of respiratory motion on tumor quantification and delineation in static PET/CT imaging," Physics in Medicine and Biology, vol. 54, no. 24, pp. 7345-7362, Dec. 2009.

[24] A. Bitarafan-Rajabi, H. Rajabi, F. Rastgou, H. Firoozabady, N. Yaghoobi, H. Malek, W. Langesteger, and M. Beheshti, "Influence of respiratory motion correction on quantification of myocardial perfusion SPECT," Journal of Nuclear Cardiology, vol. 22, no. 5, pp. 1019-1030, Oct. 2015.

[25] W. P. Segars, S. P. Mok, and B. M. W. Tsui, "Investigation of Respiratory Gating in Quantitative Myocardial SPECT," IEEE transactions on nuclear science, vol. 56, no. 1, pp. 91-96, Feb. 2009.

[26] K. Suga, Y. Kawakami, M. Zaki, T. Yamashita, T. Matsumoto, and N. Matsunaga, "Pulmonary perfusion assessment with respiratory gated 99mTc macroaggregated albumin SPECT: Preliminary results," Nuclear Medicine Communications, vol. 25, no. 2, pp. 183-193, Feb. 2004.

[27] H. Ue, H. Haneishi, H. Iwanaga, and K. Suga, "Nonlinear motion correction of respiratory-gated lung SPECT images," IEEE Transactions on Medical Imaging, vol. 25, no. 4, pp. 486-495, Apr. 2006.

[28] J.-J. Sonke, L. Zijp, P. Remeijer, and M. van Herk, "Respiratory correlated cone beam CT," Medical Physics, vol. 32, no. 4, pp. 11761186, Apr. 2005.

[29] L. Marple, "Computing the discrete-time "analytic" signal via FFT," IEEE Transactions on Signal Processing, vol. 47, no. 9, pp. 2600-2603, Sep. 1999.

[30] S. Rit, M. Vila Oliva, S. Brousmiche, R. Labarbe, D. Sarrut, and G. C. Sharp, "The Reconstruction Toolkit (RTK), an open-source cone-beam CT reconstruction toolkit based on the Insight Toolkit (ITK)," Journal of Physics: Conference Series, vol. 489, p. 012079, Mar. 2014.

[31] A. R. De Pierro, "A modified expectation maximization algorithm for penalized likelihood estimation in emission tomography," IEEE Transactions on Medical Imaging, vol. 14, no. 1, pp. 132-137, Mar. 1995.

[32] R. J. Jaszczak, K. L. Greer, C. E. Floyd, C. C. Harris, and R. E. Coleman, "Improved SPECT Quantification Using Compensation for Scattered Photons," Journal of Nuclear Medicine, vol. 25, no. 8, pp. 893-900, Aug. 1984.

[33] G. Zeng, C. Bai, and G. T. Gullberg, "A projector/backprojector with slice-to-slice blurring for efficient three-dimensional scatter modeling," IEEE transactions on medical imaging, vol. 18, pp. 722-32, Sep. 1999.

[34] D. Sarrut, M. Bardiès, N. Boussion, N. Freud, S. Jan, J.-M. Létang, G. Loudos, L. Maigne, S. Marcatili, T. Mauxion, P. Papadimitroulas, Y. Perrot, U. Pietrzyk, C. Robert, D. R. Schaart, D. Visvikis, and I. Buvat, "A review of the use and potential of the GATE Monte Carlo simulation code for radiation therapy and dosimetry applications," Medical Physics, vol. 41, no. 6, p. 064301, Jun. 2014

[35] T. Cajgfinger, S. Rit, J. M. Létang, A. Halty, and D. Sarrut, "Fixed forced detection for fast SPECT Monte-Carlo simulation," Physics in Medicine and Biology, vol. 63, no. 5, p. 055011, Mar. 2018.

[36] S. Rit, M. van Herk, L. Zijp, and J.-J. Sonke, "Quantification of the variability of diaphragm motion and implications for treatment margin construction," International Journal of Radiation Oncology, Biology, Physics, vol. 82, no. 3, pp. e399-407, Mar. 2012.

[37] Y. E. Erdi, B. W. Wessels, M. H. Loew, and A. K. Erdi, "Threshold Estimation in Single Photon Emission Computed Tomography and Planar Imaging for Clinical Radioimmunotherapy," Cancer Research, vol. 55, no. 23 Supplement, pp. 5823s-5826s, Dec. 1995.

[38] M. F. Kruis, J. B. van de Kamer, A. C. Houweling, J.-J. Sonke, J. S. A. Belderbos, and M. van Herk, "PET motion compensation for radiation therapy using a CT-based mid-position motion model: Methodology and clinical evaluation," International Journal of Radiation Oncology, Biology, Physics, vol. 87, no. 2, pp. 394-400, Oct. 2013.

[39] S.-J. Park, D. Ionascu, J. Killoran, M. Mamede, V. H. Gerbaudo, L. Chin, and R. Berbeco, "Evaluation of the combined effects of target size, respiratory motion and background activity on $3 \mathrm{D}$ and $4 \mathrm{D}$ PET/CT 
images," Physics in Medicine and Biology, vol. 53, no. 13, pp. 36613679, Jun. 2008.

[40] T. Li and L. Xing, "Optimizing 4D cone-beam CT acquisition protocol for external beam radiotherapy," International Journal of Radiation Oncology, Biology, Physics, vol. 67, no. 4, pp. 1211-1219, Mar. 2007.

[41] S. A. Nehmeh, Y. E. Erdi, T. Pan, A. Pevsner, K. E. Rosenzweig, E. Yorke, G. S. Mageras, H. Schoder, P. Vernon, O. Squire, H. Mostafavi, S. M. Larson, and J. L. Humm, "Four-dimensional (4D) PET/CT imaging of the thorax," Medical Physics, vol. 31, no. 12, pp. 31793186, Dec. 2004.

[42] T. Li, B. Thorndyke, E. Schreibmann, Y. Yang, and L. Xing, "Modelbased image reconstruction for four-dimensional PET: 4D PET reconstruction," Medical Physics, vol. 33, no. 5, pp. 1288-1298, Apr. 2006.

[43] R. Manjeshwar, Xiaodong Tao, E. Asma, and K. Thielemans, "Motion Compensated Image Reconstruction of Respiratory Gated PET/CT," in 3rd IEEE International Symposium on Biomedical Imaging: Macro to Nano, 2006. Arlington, Virginia, USA: IEEE, 2006, pp. 674-677.

[44] Z. Lu, G. Chen, K.-H. Lin, T.-H. Wu, and G. S. P. Mok, "Evaluation of Different CT Maps for Attenuation Correction and Segmentation in Static 99mTc-MAA SPECT/CT for 90Y Radioembolization Treatment Planning - a Simulation Study," Medical Physics, vol. n/a, no. n/a, 2021.

[45] M. M. Osman, C. Cohade, Y. Nakamoto, and R. L. Wahl, "Respiratory motion artifacts on PET emission images obtained using CT attenuation correction on PET-CT," European Journal of Nuclear Medicine and Molecular Imaging, vol. 30, no. 4, pp. 603-606, Apr. 2003.

[46] G. W. Goerres, C. Burger, E. Kamel, B. Seifert, A. H. Kaim, A. Buck, T. C. Buehler, and G. K. von Schulthess, "Respiration-induced Attenuation Artifact at PET/CT: Technical Considerations," Radiology, vol. 226, no. 3, pp. 906-910, Mar. 2003.

[47] A. G. Pitman, V. Kalff, B. V. Every, B. Risa, L. R. Barnden, and M. J. Kelly, "Effect of Mechanically Simulated Diaphragmatic Respiratory Motion on Myocardial SPECT Processed With and Without Attenuation Correction," Journal of Nuclear Medicine, vol. 43, no. 9, pp. 1259-1267, Sep. 2002.

[48] D. Zhang, B.-H. Yang, N. Y. Wu, and G. S. P. Mok, "Respiratory average CT for attenuation correction in myocardial perfusion SPECT/CT," Annals of Nuclear Medicine, vol. 31, no. 2, pp. 172-180, Feb. 2017.

[49] F. Pönisch, C. Richter, U. Just, and W. Enghardt, "Attenuation correction of four dimensional (4D) PET using phase-correlated 4D-computed tomography," Physics in Medicine and Biology, vol. 53, no. 13, pp. N259-268, Jul. 2008.

[50] D. Zhang, M. Ghaly, and G. S. P. Mok, "Interpolated CT for attenuation correction on respiratory gating cardiac SPECT/CT - A simulation study," Medical Physics, vol. 46, no. 6, pp. 2621-2628, 2019.

[51] L. Shi, J. A. Onofrey, H. Liu, Y.-H. Liu, and C. Liu, "Deep learningbased attenuation map generation for myocardial perfusion SPECT," European Journal of Nuclear Medicine and Molecular Imaging, vol. 47, no. 10, pp. 2383-2395, Sep. 2020. 